\title{
Lungenarterienembolie
}

\section{Pulmonary embolism}

\author{
S. Sudarski, T. Henzler
}

\section{Übersicht \\ Hintergrund \\ Bildgebung \\ LE-Diagnostik mittels CTPA \\ LE-Diagnostik bei Schwangeren}

entscheidend. Ziel ist es, so schnell wie möglich eine Therapie einzuleiten oder eine LE zuverlässig auszuschließen. Die Computertomografie der Pulmonalarterien (CTPA) mit Mehrzeilen-CT-Systemen ist der Goldstandard im diagnostischen Vorgehen. Darüber hinaus kann die CTPA weitere relevante Informationen liefern, z. B., ob eine Rechtsherzbelastung vorliegt. Es gibt eine ganze Reihe von Scan- und Kontrastmittelprotokollen,

\section{Zusammenfassung}

Eine Lungenarterienembolie (LE) erfordert einen schnellen Diagnosealgorithmus, da sie unbehandelt mit einer hohen Morbidität und Mortalität einhergeht. Für das diagnostische Vorgehen sind die initiale klinische Wahrscheinlichkeit einer LE und das individuelle Risikoprofil des Patienten

\section{Hintergrund}

\section{Definition}

Die Lungenarterienembolie (LE) ist ein akutes, potenziell lebensbedrohliches Krankheitsbild. Hierbei kommt es zu einem teilweisen oder völligen Verschluss eines oder mehrerer Segmente der Pulmonalarterien, meist durch fortgeleitete Thromben aus den tiefen Beinvenen oder Beckenvenen, also als Folge einer tiefen Venenthrombose (TVT). Die Erkrankungen LE und TVT fallen unter den Überbegriff der sog. venösen Thromboembolie (VTE).

\section{Risikofaktoren}

Eine LE wird durch eine ganze Reihe verschiedener Risikofaktoren begünstigt, die deckungsgleich sind mit denen einer VTE im Allgemeinen. Diese Faktoren kann man unterteilen in die - meist permanenten - patientenbezogenen Risikofaktoren und die - meist temporären - situationsbezogenen Risikofaktoren [1]. Zu den situationsbezogenen Faktoren zählen stattgehabte

Operationen, Traumata, Immobilisation, Schwangerschaft, Einnahme oraler Kontrazeptiva oder eine Hormontherapie in einem Zeitfenster zwischen 6 Wochen und 3 Monaten vor dem Ereignis. Das höchste Risiko bei Schwangeren besteht im 3. Trimester, wobei eine durchgeführte In-vitro-Fertilisation das Risiko der Schwangeren noch zusätzlich steigert [1]. Innerhalb der ersten 6 Wochen nach Entbindung ist das Risiko einer LE ebenfalls erhöht. Auch onkologische Erkrankungen sind anerkannte Risikofaktoren einer LE, wobei nicht alle Malignome als gleichermaßen prädisponierend gelten: Das höchste Risiko haben hämatoonkologische Patienten, Patienten mit Bronchialkarzinomen, gastrointestinalen Tumoren, Pankreastumoren und Hirntumoren [1].

Situationsbezogene Risikofaktoren sind u. a. stattgehabte Operationen, Traumata, Immobilisation, Schwangerschaft, orale Kontrazeptiva, Hormontherapien oder Tumoren. 


\section{Epidemiologie}

Epidemiologische Daten zur Inzidenz von Lungenembolien sind mit der Einschränkung zu sehen, dass viele akute Lungenarterienembolien asymptomatisch verlaufen und somit unerkannt bleiben. Die meisten Daten basieren auf der Häufigkeit der Diagnose der VTE im Allgemeinen und die jährliche Inzidenz dieses Krankheitsbildes wird mit 100 - 200 pro 100000 Einwohnern beziffert. Somit ist es die dritthäufigste kardiovaskuläre Erkrankung. Die Rate an Patienten, die an einer akuten Lungenembolie oder an den Folgen einer VTE versterben, ist noch schwieriger zu beziffern, und die in der Literatur publizierten Zahlen sind wahrscheinlich viel zu niedrig. Auf Basis epidemiologischer Berechnungsmodelle mit Zahlen der Todesursachenregister aus 6 Ländern der Europäischen Union ist davon auszugehen, dass allein bei rund $60 \%$ der Patienten, die an den Folgen einer VTE starben (Patienten, die an einer akuten LE verstarben ausgenommen), die Diagnose einer LE vor dem Tod nie gestellt worden war [1].

\section{Klinik}

Weil die klinischen Symptome unspezifisch sind oder vollständig fehlen, bleibt eine LE häufig unerkannt. Das Mortalitätsrisiko eines Patienten hängt glücklicherweise jedoch mit der klinischen Präsentation zusammen: Man unterscheidet Patienten mit einer „HighRisk“-LE (Schock oder Hypotonie) von denen mit einer „Non-High-Risk“-LE (kein Schock oder Hypotonie). Beide Patientengruppen können noch eine Vielzahl anderer Symptome aufweisen, am häufigsten Dyspnoe, Tachykardie, Schwindel, Thoraxschmerz oder Zustand nach Synkope. Außerdem kommen Hämoptysen oder Zyanosen vor oder aber auch lediglich Husten oder Fieber. In der Blutgasanalyse findet sich - stark abhängig von Begleiterkrankungen - zuweilen auch eine Hypoxämie oder eine Hypokapnie. EKG-Veränderungen wie die $S_{1} / Q_{3}$-Konfiguration, ein P-pulmonale oder ein Rechtslagetyp können einen indirekten Hinweis auf eine LE mit Rechtsherzbelastung geben.

\section{Outcome}

Mortalität. Eine korrekte Einschätzung des klinischen Schweregrades der vermuteten LE und ein nachfolgender korrekter schneller Diagnose- und Therapiealgorithmus sind im Hinblick auf die 30-Tage-Mortalität prognostisch entscheidend [1]. Bei sehr hoher klinischer Wahrscheinlichkeit sollte die spezifische
Therapie schon eingeleitet werden, bevor die definitive Diagnose gestellt wurde.

CTEPH. Patienten, die einmal eine LE hatten, neigen zu Rezidiv-Embolien. Bei diesen Patienten besteht die Gefahr, dass sich eine pulmonale Hypertonie entwickelt (chronische thromboembolische pulmonale Hypertonie $=$ CTEPH). Zwei Jahre nach Diagnose einer LE wird die Inzidenz hierfür in der Literatur mit ca. $4 \%$ beziffert [2].

Rechtsherzbelastung. Eine Rechtsherzbelastung, die bei Patienten im Rahmen der LE auftrat, kann sich verschlechtern und geht dann mit einem erhöhten Risiko kardialer Ereignisse einher.

\section{Risikoeinschätzung}

Klinische Wahrscheinlichkeit. Da die klinischen Symptome einer akuten LE häufig unspezifisch sind, schätzt man ihre klinische Wahrscheinlichkeit mit Scores, bei denen die o.g. patienten- und situationsbezogenen Risikofaktoren maßgeblich sind. Beim revidierten Wells-Score und beim Geneva-Score kann man in niedrige, mittlere oder hohe Prätestwahrscheinlichkeit für eine LE unterscheiden oder weiter vereinfacht beim revidierten zweiseitigen Score entweder in „LE wahrscheinlich“ oder „LE unwahrscheinlich“ [1]. Abhängig von der so definierten Prätestwahrscheinlichkeit folgt anschließend die weitere Diagnostik (Abb.1).

D-Dimer-Test. Bei der als Folge eines Gerinnungsprozesses erhöhten Fibrinolyseaktivität im Blut können vermehrt D-Dimere - Abbauprodukte der Fibrinolyse nachgewiesen werden. Allgemein kann man sagen, dass ein negativer D-Dimer-Test bei leitliniengerechter Anwendung des Tests eine LE sicher ausschließt, also einen hohen negativen prädiktiven Wert aufweist. Umgekehrt ist der positive prädiktive Wert jedoch niedrig, weil der Test bei Patienten mit onkologischer Grunderkrankung, bei hospitalisierten Patienten und bei Schwangeren oft positiv ist. Üblicherweise wird ab einem Cut-off-Wert von $500 \mu \mathrm{g} / \mathrm{l}$ von einem positiven D-Dimer-Test gesprochen, jedoch finden zunehmend altersangepasste Schwellenwerte Verwendung [3], und es muss zwischen moderat sensitiven und hochsensitiven Testverfahren unterschieden werden (Abb.1). So findet sich in den aktuellen Leitlinien zur Lungenemboliediagnostik die Empfehlung (s.a. Tab. 1) zur weiteren Diagnostik, wenn ein moderat sensitiver D-Dimer-Test bei einem Patienten mit mittlerer Prätestwahrscheinlichkeit für eine LE negativ ist. Gar nicht empfohlen wird ein D-Dimer-Test bei Patienten mit 


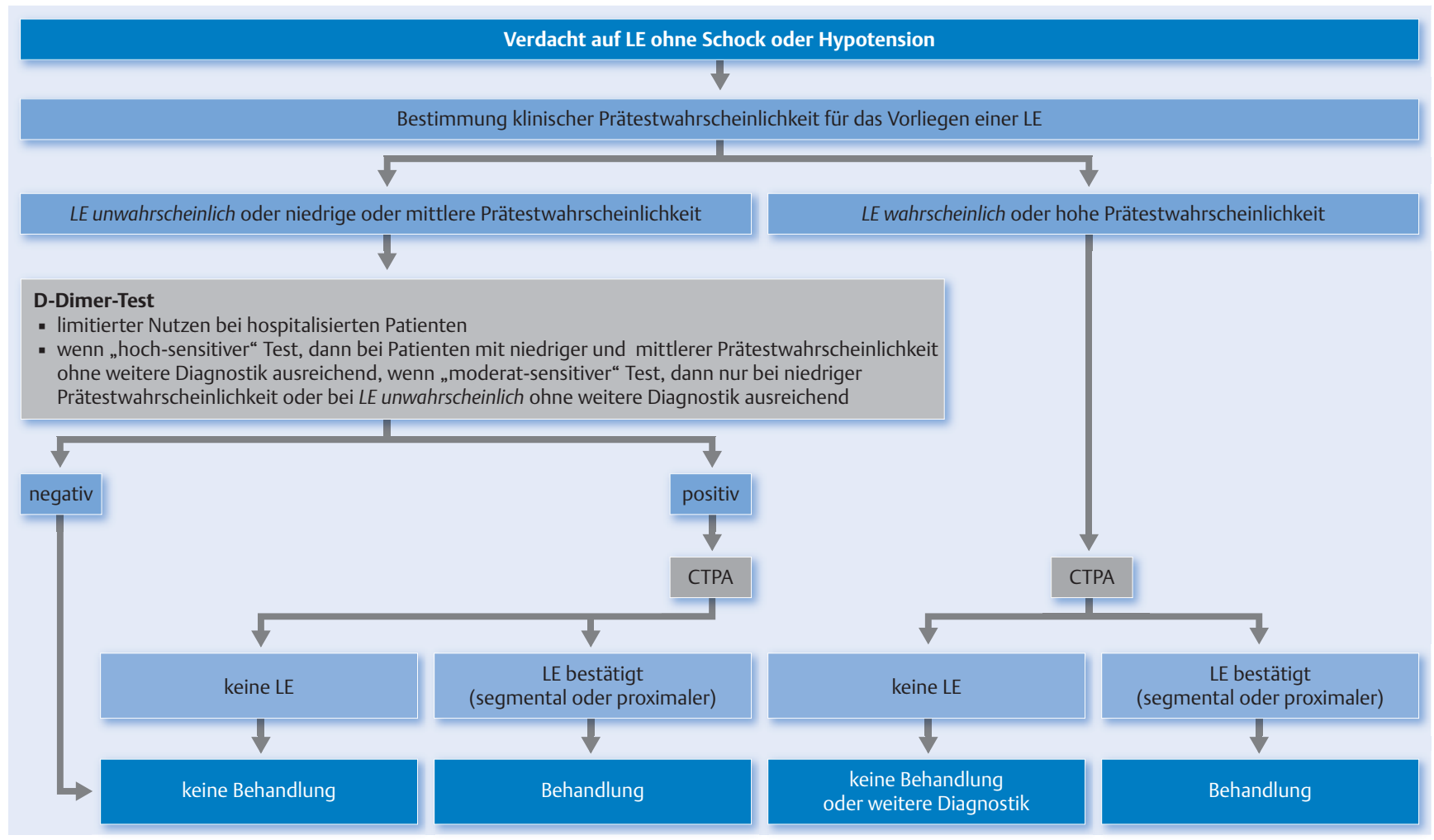

Abb. 1 Diagnosealgorithmus für Patienten mit Verdacht auf eine LE ohne Schock oder Hypotonie (nach ESC-Guidelines 2014 [1]).

hoher Prätestwahrscheinlichkeit für eine LE (s.a. Tab.1, Nicht invasive Bildgebung

Abb.1), da normale D-Dimer-Werte in dieser Patientengruppe eine LE nicht einmal bei hochsensitiven Assays definitiv ausschließen können.

Ein D-Dimer-Test ist nur bei Patienten mit einer niedrigen oder mittleren Prätestwahrscheinlichkeit für eine LE sinnvoll. Bei Patienten mit mittlerer Prätestwahrscheinlichkeit sollte dabei idealerweise ein hochsensitiver Assay verwendet werden.

\section{Bildgebung}

\section{Invasive Bildgebung: Pulmonalisangiografie}

Die Katheterangiografie der Pulmonalarterien (Pulmonalisangiografie, PA) ist der historische Goldstandard für die Diagnostik der LE. Die PA ist heute jedoch durch die nicht invasive Bildgebung ersetzt worden. Ihr Einsatzgebiet beschränkt sich daher diagnostisch auf Patienten mit Verdacht auf eine chronische thromboembolische pulmonale Hypertonie und therapeutisch auf Patienten, bei denen eine kathetergesteuerte Thrombolyse indiziert ist [4].

\section{CT-Angiografie}

Die CT-Angiografie (CTA) der Pulmonalarterien (CTPA) wird in den ESC-Leitlinien von 2014 - immer noch in Bezug auf die PIOPED-II-Studie, die primär Patienten an 4-Zeilen-CTs eingeschlossen hatte - als diagnostische Modalität der Wahl bei Patienten mit Verdacht auf eine LE empfohlen [1]. Hierbei sind der positive und der negative prädiktive Wert der CT-Diagnose immer differenziert im Hinblick auf die Prätestwahrscheinlichkeit des Patienten zu betrachten:

- Bei Patienten mit niedriger bzw. mittlerer Wahrscheinlichkeit einer LE auf Basis des Wells-Scores hatte die CT einen hohen negativen prädiktiven Wert von 96\% bzw. 89\%. Bei Patienten mit hoher Prätestwahrscheinlichkeit sank dieser Wert auf $60 \%$.

- Genau konträr stellten sich die Ergebnisse für den positiven prädiktiven Wert dar: Bei Patienten mit mittlerer oder hoher Prätestwahrscheinlichkeit für eine LE waren die Prozentzahlen hoch (92-96\%), bei Patienten mit niedriger Prätestwahrscheinlichkeit dagegen bedeutend niedriger (58\%). 
Tabelle 1

Diagnoseempfehlungen bei Verdacht auf LE nach den ESC-Leitlinien 2008 vs. 2014.

\begin{tabular}{|c|c|c|c|c|c|c|}
\hline \multirow{2}{*}{$\begin{array}{l}\text { Modalität/ } \\
\text { Verfahren }\end{array}$} & \multirow[t]{2}{*}{ Diagnoseempfehlungen } & \multicolumn{3}{|l|}{2008} & \multicolumn{2}{|l|}{2014} \\
\hline & & Klasse & Level & & Klasse & Level \\
\hline \multicolumn{7}{|c|}{ Verdacht auf High-Risk-LE (mit Schock oder Hypotonie) } \\
\hline $\begin{array}{l}\text { CTPA und } \\
\text { TTE }\end{array}$ & $\begin{array}{l}\text { bei Verdacht auf High-Risk-LE Notfall-CTPA oder Bedside-TTE (abhängig von Verfüg- } \\
\text { barkeit und klinischen Umständen) }\end{array}$ & 1 & $\mathrm{C}$ & $=$ & 1 & $\mathrm{C}$ \\
\hline $\begin{array}{l}\text { CUS oder } \\
\text { TEE }\end{array}$ & $\begin{array}{l}\text { bei Patienten mit Verdacht auf High-Risk-LE und Zeichen der RVD, die zu instabil sind } \\
\text { für die Durchführung der CTPA, sollte am Krankenbett nach venösen oder pulmonal- } \\
\text { arteriellen Thromben gesucht werden CUS und/oder TEE, falls sofort verfügbar }\end{array}$ & - & - & + & $2 b$ & $\mathrm{C}$ \\
\hline PA & $\begin{array}{l}\text { PA bei Patienten, die instabil waren und direkt ins Katheterlabor gegangen sind, im } \\
\text { Falle eines Ausschlusses eines akuten Koronarsyndroms }\end{array}$ & - & - & + & $2 b$ & $\mathrm{C}$ \\
\hline \multicolumn{7}{|c|}{ Verdacht auf Non-High-Risk-LE (ohne Schock oder Hypotonie) } \\
\hline & $\begin{array}{l}\text { es sollen validierte Kriterien zur LE-Diagnose genutzt werden bei Patienten mit Verdacht } \\
\text { auf LE ohne Schock oder Hypotonie }\end{array}$ & 1 & $\mathrm{~B}$ & $=$ & 1 & B \\
\hline & $\begin{array}{l}\text { Diagnosestrategie auf Basis klinischer Wahrscheinlichkeit (entweder Klinik oder validierte } \\
\text { PTW-Kriterien) }\end{array}$ & 1 & A & $=$ & 1 & A \\
\hline D-Dimere & $\begin{array}{l}\text { D-Dimer-Test (vorzugsweise „high-sensitive“) bei ambulanten Patienten mit niedriger } \\
\text { oder mittlerer PTW oder „LE unwahrscheinlich“, um unnötige Bildgebung zu vermeiden }\end{array}$ & 1 & A & $=$ & 1 & A \\
\hline D-Dimere & $\begin{array}{l}\text { Ausschluss LE durch negativen D-Dimer-Test („high-sensitive“ und „moderat sensitiv“) } \\
\text { bei Patienten mit niedriger PTW oder „LE unwahrscheinlich“ }\end{array}$ & 1 & A & $=$ & 1 & A \\
\hline D-Dimere & weitere Diagnostik bei negativem moderat sensitivem D-Dimer-Test bei mittlerer PTW & $2 b$ & $\mathrm{C}$ & & $2 b$ & B \\
\hline D-Dimere & $\begin{array}{l}\text { kein Test bei Patienten mit hoher PTW, da normale D-Dimer-Werte nicht mal bei } \\
\text { „high-sensitive“ Assays eine LE definitiv ausschließen können }\end{array}$ & 3 & $\mathbf{C}$ & & 3 & B \\
\hline CTPA & $\begin{array}{l}\text { sicherer Ausschluss einer LE mittels CTPA bei Patienten mit niedriger oder mittlerer } \\
\text { PTW oder „PE unwahrscheinlich“ }\end{array}$ & 1 & A & $=$ & 1 & A \\
\hline CTPA & $\begin{array}{l}\text { sicherer Ausschluss einer LE bei Patienten mit hoher PTW oder „LE wahrscheinlich“ } \\
\text { möglich statt }\end{array}$ & & & + & $2 a$ & B \\
\hline CTPA & weitere Diagnostik bei Ausschluss LE bei Patienten mit hoher PTW & $2 \mathrm{a}$ & B & - & & \\
\hline CTPA & segmentaler oder proximalerer Thrombus sichert Diagnose LE & 1 & A & & 1 & B \\
\hline CTPA & $\begin{array}{l}\text { weitere Diagnostik zur Diagnosesicherung LE bei isoliert subsegmentalen Clots kann } \\
\text { erfolgen statt }\end{array}$ & & & + & $2 b$ & $C$ \\
\hline CTPA & $\begin{array}{l}\text { weitere Diagnostik zur Diagnosesicherung LE bei isoliert subsegmentalen Clots sollte } \\
\text { erfolgen bei Patienten mit niedriger PTW }\end{array}$ & $2 a$ & B & - & & \\
\hline CTPA & $\begin{array}{l}\text { weitere Diagnostik zur Diagnosesicherung LE bei isoliert subsegmentalen Clots kann } \\
\text { erfolgen bei Patienten mit mittlerer PTW }\end{array}$ & $2 b$ & $B$ & - & & \\
\hline CTPA & $\begin{array}{l}\text { weitere Diagnostik zur Diagnosesicherung LE bei isoliert subsegmentalen Clots kann } \\
\text { erfolgen bei Patienten mit hoher PTW }\end{array}$ & $2 b$ & B & - & & \\
\hline
\end{tabular}


Tabelle 1

(Fortsetzung)

\begin{tabular}{|c|c|c|c|c|c|c|}
\hline \multirow{2}{*}{$\begin{array}{l}\text { Modalität/ } \\
\text { Verfahren }\end{array}$} & \multirow[t]{2}{*}{ Diagnoseempfehlungen } & \multicolumn{3}{|l|}{2008} & \multicolumn{2}{|l|}{2014} \\
\hline & & Klasse & Level & & Klasse & Level \\
\hline $\begin{array}{l}\text { V/P-Szinti- } \\
\text { grafie }\end{array}$ & Ausschluss LE bei normaler Perfusionsszintigrafie und & & & + & 1 & A \\
\hline $\begin{array}{l}\text { V/P-Szinti- } \\
\text { grafie }\end{array}$ & Diagnose LE durch „high-probability“ V/P-Szintigrafie statt & & & + & $2 a$ & B \\
\hline $\begin{array}{l}\text { V/P-Szinti- } \\
\text { grafie }\end{array}$ & $\begin{array}{l}\text { Ausschluss LE bei normaler Perfusionsszintigrafie bei Patienten mit niedriger oder } \\
\text { mittlerer PTW }\end{array}$ & 1 & A & - & & \\
\hline \multirow[t]{2}{*}{$\begin{array}{l}\text { V/P-Szinti- } \\
\text { grafie }\end{array}$} & $\begin{array}{l}\text { mögliche Diagnosestellung LE durch „high-probability“ V/P-Szintigrafie bei Patienten } \\
\text { mit niedriger PTW aber ... }\end{array}$ & $2 a$ & B & - & & \\
\hline & weitere Diagnostik bei ausgewählten Patienten möglich zur Diagnosesicherung & $2 b$ & B & - & & \\
\hline $\begin{array}{l}\text { V/P-Szinti- } \\
\text { grafie }\end{array}$ & Diagnose LE durch „high-probability“ V/P-Szintigrafie bei Patienten mit hoher PTW & 1 & A & - & & \\
\hline $\begin{array}{l}\text { V/P-Szinti- } \\
\text { grafie } \\
\text { CUS }\end{array}$ & $\begin{array}{l}\text { möglicher LE-Ausschluss mit nicht diagnostischem V/P-Scan plus negativem proximalem } \\
\text { CUS bei Patienten mit niedriger PTW oder „PE unwahrscheinlich“ }\end{array}$ & $2 a$ & B & $=$ & $2 a$ & B \\
\hline $\begin{array}{l}\text { V/P-Szinti- } \\
\text { grafie }\end{array}$ & $\begin{array}{l}\text { weitere Diagnostik bei nicht diagnostischem V/P-Scan bei Patienten mit mittlerer PTW } \\
\text { zur Diagnose/zum Ausschluss LE }\end{array}$ & 1 & B & - & & \\
\hline $\begin{array}{l}\text { Unterschen- } \\
\text { kel-CuS }\end{array}$ & $\begin{array}{l}\text { möglich bei ausgewählten Patienten mit Verdacht auf LE, um weitere Diagnostik zu } \\
\text { umgehen, wenn positiv }\end{array}$ & $2 b$ & B & $=$ & $2 \mathrm{~b}$ & B \\
\hline CUS & Diagnose LE bei proximaler TVT bei Patienten mit Verdacht auf LE & 1 & $\mathrm{~B}$ & $=$ & 1 & B \\
\hline CUS & weitere Diagnostik bei lediglich distaler TVT zur Diagnose LE statt & & & + & $2 a$ & B \\
\hline CUS & $\begin{array}{l}\text { weitere Diagnostik bei lediglich distaler TVT zur Diagnose LE bei niedriger und } \\
\text { mittlerer PTW }\end{array}$ & $2 a$ & B & - & & \\
\hline CUS & weitere Diagnostik bei lediglich distaler TVT zur Diagnose LE bei hoher PTW & $2 b$ & B & - & & \\
\hline PA & bei Diskrepanzen zwischen klinischer Evaluation und nicht invasiver Bildgebung & $2 a$ & C & & $2 b$ & $C$ \\
\hline MRA & sollte nicht zum Ausschluss LE genutzt werden & & & + & 3 & A \\
\hline
\end{tabular}

CUS= Kompressionsultraschall; MRA= MR-Angiografie; PA = Pulmonalisangiografie; TEE = transösophageale Echokardiografie; TTE= transthorakale Echokardiografie, PTW= Prätestwahrscheinlichkeit; + wenn neue Empfehlung; - wenn gestrichene Empfehlung; =wenn gleichbleibende Empfehlung; Änderungen von Klasse/Level fett markiert 
Neuere Arbeiten plädieren für die CTPA als alleinige Untersuchung zum LE-Ausschluss: In prospektiven Studien war die Rate thromboembolischer Ereignisse innerhalb von 3 Monaten nach einer negativen CTPA niedrig (zwischen 0,3\% und 1,5\%) - und zwar unabhängig von der initialen Prätestwahrscheinlichkeit [5-7].

\section{- CT-Venografie}

Die in der PIOPED-II-Studie untersuchte Kombination aus CTPA und CTV erhöhte die Anzahl nicht eindeutiger Befunde (von 6\% bei CTPA als alleiniger Diagnostik hin zu 10,5\% bei der Kombination aus CTPA und CTV). Die Sensitivität nahm zwar durch eine ergänzende CTV von $83 \%$ auf $90 \%$ zu, die Spezifität blieb jedoch unverändert (95\%) [8]. Ein weiteres Ergebnis der PIOPED-II-Studie war die Beobachtung, dass mit der CTV bei $3 \%$ der untersuchten Patienten Emboli in der V. cava inferior und den Beckenvenen entdeckt wurden, und dass alle diese Patienten eine LE in der CTPA aufwiesen. Obwohl die Zunahme bei der Sensitivität nicht statistisch signifikant war, schlossen die PIOPED-II-Autoren aus den erhobenen Daten, dass die CTPA alleine aufgrund ihrer Rate an falsch negativen Befunden von 17\% nicht geeignet sei, um eine LE zuverlässig auszuschließen, sondern dass dafür die Kombination mit der CTV nötig sei. Die European Society of Cardiology (ESC) folgerte aus den gleichen Daten genau das Gegenteil, nämlich, dass eine Kombination aus CTPA und CTV nur einen marginalen diagnostischen Mehrwert bei gleichzeitig signifikant höherer Strahlenbelastung aufweist. Die Fleischner Society blieb weniger restriktiv und empfahl - wieder auf der Basis der PIOPED-II-Daten - die zusätzliche CTV in Abwägung der Wichtigkeit einer umfassenden Erhebung des Gefäßstatus und das Ausweichen auf den Ultraschall der unteren Extremität bei strahlenhygienischen Bedenken.
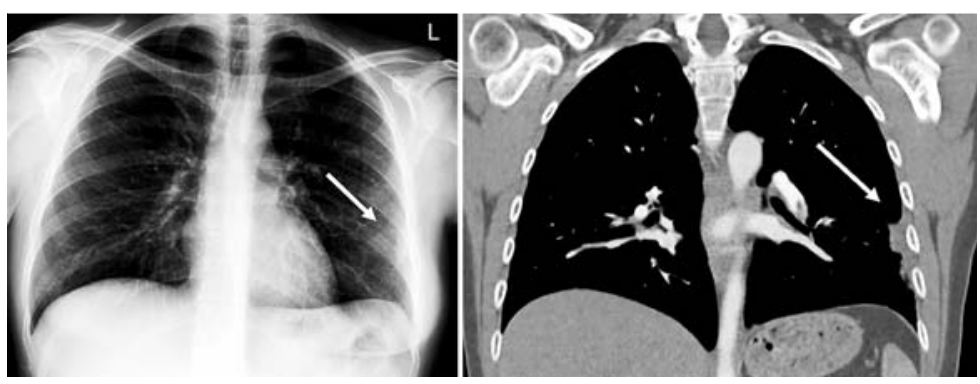

Abb. 2 Lungeninfarkttypisches pleuraständiges keilförmiges Infiltrat bei einem Patienten mit LE im konventionellen Röntgen-Thorax („hampton hump“) und in der korrespondierenden CTPA.
In der Literatur herrscht große Uneinigkeit darüber, ob der zusätzliche diagnostische Benefit der CTVenografie (CTV) die längere Untersuchungszeit, die höheren Ausgaben und die größere Strahlenbelastung rechtfertigt.

\section{Ventilations/Perfusions-Szintigrafie}

Die Ventilations/Perfusions-Szintigrafie hat eine Sensitivität von ca. $78 \%$ bei einer hohen Spezifität von ca. $98 \%$, sodass eine LE im Fall einer negativen Szintigrafie ebenfalls ausgeschlossen ist und keine weiteren therapeutischen Maßnahmen indiziert sind [6,9]. Als Vorteil wird häufig die relativ niedrige Strahlendosis (um die 1,1 mSv) angesehen - insbesondere im Hinblick auf die weiblichen Brustdrüsen bei der Untersuchung von Frauen. Mit der Weiterentwicklung der CT-Scanner verpufft dieser Vorteil jedoch mehr und mehr [10]. Hinzu kommen gravierende Einschränkungen der Methode im Hinblick auf Verfügbarkeit im Nachtdienst und am Wochenende. Ein weiteres Problem ist die hohe Quote von zwei Drittel nicht eindeutigen Diagnosen, die sich auch in der Befundung niederschlägt ( Normalbefund = keine LE, LE wahrscheinlich = positiver Nachweis oder nicht diagnostischer Scan).

Insgesamt ist mit dieser Methode im Optimalfall lediglich ein indirekter Nachweis der LE möglich.

\section{- SPECT}

Die nuklearmedizinische Methode der Single-Photonen-Emissions-CT (SPECT) spielt in den europäischen Leitlinien keine Rolle bei der Diagnostik der LE.

\section{MR-Angiografie}

Die kontrastmittelunterstützte Untersuchung der Pulmonalarterien mittels MR-Angiografie (MRA) ist in verschiedenen Studien mit teilweise vielversprechenden Ergebnissen evaluiert worden [11]. Jedoch konnten diese Ergebnisse nicht flächendeckend reproduziert werden, sodass die kontrastmittelunterstützte MRAngiografie in den ESC-Leitlinien von 2014 explizit nicht für den Ausschluss einer Lungenembolie empfohlen wird (Tab. 1). Für den Einsatz von nicht kontrastmittelgestützten MRA-Techniken existieren aufgrund der schwachen Evidenz gar keine Empfehlungen.

\section{- Thorax-Röntgen}

Das konventionelle Röntgen spielt keine diagnostische Rolle in den hier primär als Referenz dienenden ESCLeitlinien für die Lungenembolie und gibt mit geringer Sensitivität und Spezifität lediglich indirekte Hinweise auf eine Rechtsherzbelastung oder kann (Abb.2) pleuraständige infarkttypische keilförmige Verschattungen 
zeigen. Der mögliche Einsatz bei Schwangeren als initiale Bildgebung nach nicht eindeutiger Ultraschalluntersuchung zum Ausschluss einer TVT vor Durchführung einer Szintigrafie ist in den Empfehlungen der ACR aufgeführt [12].

In den revidierten Diagnoseempfehlungen 2014 der ESC für die akute LE ist erstmals schriftlich verankert, dass die MR-Angiografie nicht zum LE-Ausschluss genutzt werden sollte.

\section{LE-Diagnostik mittels CTPA}

\section{Bedeutung der CTPA}

Vorteile. Seit Einführung der Mehrzeilen-Spiral-CT wurden die CT-Scanner kontinuierlich weiterentwickelt im Hinblick auf eine verbesserte räumliche und zeitliche Auflösung und eine niedrigere Strahlenbelastung. Vorteile der CTPA-Diagnostik sind ihre breite Verfügbarkeit und einfache Durchführbarkeit auch in Nacht- und Wochenenddiensten. Neben dem direkt erbrachten Nachweis der Emboli/des Embolus kann eine eventuelle Rechtsherzbelastung diagnostiziert und die Prognose eingeschätzt werden (s. u.) [13-15]. Außerdem können Differenzialdiagnosen je nach verwendetem Kontrastmittelprotokoll in einer Art OneStop-Shop-Verfahren mitabgebildet werden (s.u.).

Aussagen. Die Sensitivität und die Spezifität der CTPA zum Nachweis einer LE liegen mit aktuell verfügbaren CT-Systemen (>4-Zeilen-CT) bei nahezu 100\%. Der hohe negative prädiktive Wert besagt, dass eine Lungenembolie sicher ausgeschlossen werden kann und keine weitere Diagnostik neben der CTPA notwendig ist [5]. Wird eine LE nachgewiesen, ist es im Hinblick auf die therapeutischen Konsequenzen auf Basis der Datenlage unstrittig, dass bei einer zentralen (Abb.3) oder einer segmentalen LE mit oder ohne Nachweis einer tiefen Beinvenenthrombose der Nutzen einer Therapie die Risiken überwiegt.

Isolierte subsegmentale akute LE. Hierbei gilt es jedoch zu beachten, dass die Datenlage für eine isolierte subsegmentale akute LE in der CTPA nicht eindeutig ist. Der Verdacht auf eine solche isolierte subsegmentale LE ergibt sich in annähernd 10\% (5,5-14,2\%) aller Mehrzeilen-CTPA-Untersuchungen, wobei diese Befunde jedoch einen niedrigen positiven prädiktiven Wert haben und eine schlechte Inter-Reader-Übereinstimmung aufweisen [16, 17]. Dementsprechend urteilen die ESC-Leitlinien sehr zurückhaltend, dass im Fall

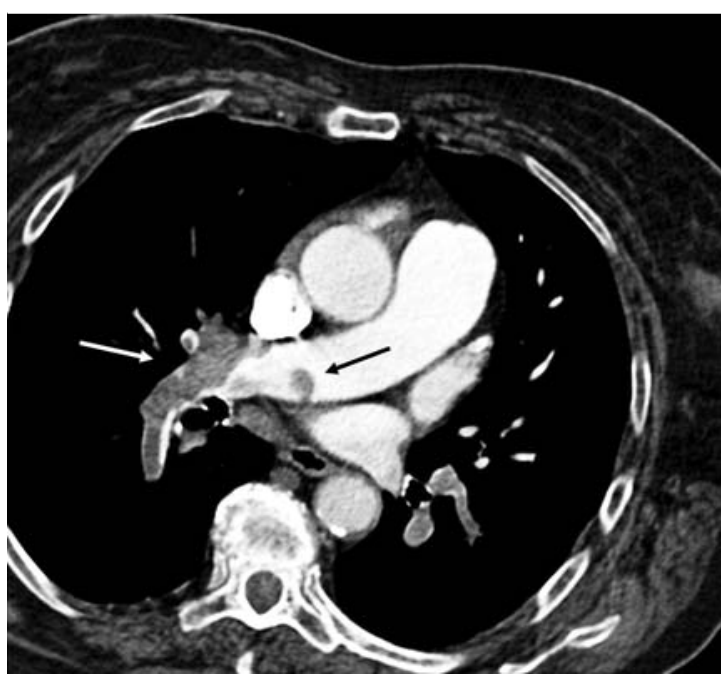

Abb. 3 CTPA mit Nachweis einer zentralen Lungenembolie und okkludierend emboligenem Material in der rechten Pulmonalarterie.

einer isolierten subsegmentalen LE in der CTPA eine tiefe Beinvenenthrombose definitiv ausgeschlossen werden sollte, und bei gegebenem Ausschluss dann die Entscheidung für oder gegen eine Therapie unter Zuhilfenahme der klinischen Prätestwahrscheinlichkeit und des individuellen Blutungsrisikos individuell gefällt werden sollte.

Insgesamt erfährt das Verfahren der CTPA in der Aktualisierung der ESC-Diagnoseempfehlungen von 2008 auf 2014 (Tab. 1) eine weitere Stärkung besonders bei Patienten mit hoher Prätestwahrscheinlichkeit für eine LE.

\section{Praktische Durchführung}

Eine Untersuchung mit diagnostischer Bildqualität ist die Grundvoraussetzung, um eine LE zuverlässig auszuschließen oder nachzuweisen. Hierfür ist die Auswahl eines passenden Scan-/Kontrastmittelprotokolls ebenso entscheidend wie die Patientenführung, die mit der sicheren Anlage eines ausreichend großen venösen Zugangs und die Aufklärung über die Dauer der Untersuchung beginnt, das sichere Anleiten von Atemkommandos einschließt und mit der Aufklärung über das plötzliche Hitzegefühl bei Kontrastmittelinjektion endet. Im Folgenden wird auf die technische Durchführung und besonders Artefakte durch falsche Atmung näher eingegangen. 


\section{Datenakquisition}

Geräte. Generell gilt, dass Geräte mit mehr als 4 Zeilen für eine Lungenemboliediagnostik geeignet sind. Ab 64 Zeilen gelingt die Untersuchung erfahrungsgemäß auch zuverlässig bei Patienten mit Dyspnoe und nur sehr kurzer Atemanhaltephase.

Kollimation, Rotationszeit, Pitch. Bei allen CT-Scannern sollten eine möglichst dünne Kollimation ( $\leq 1 \mathrm{~mm})$, eine möglichst kurze Rotationszeit $(\leq 0,5 \mathrm{~s})$ und ein hoher Pitch (>1) gewählt werden, um einen für die Lungenemboliediagnostik bestmöglichen Kompromiss zwischen räumlicher Auflösung und Scandauer zu erreichen.

Röhrenspannung und -strom. Für die Bildqualität sind außerdem die Wahl von Röhrenspannung $(\mathrm{kV})$ und Röhrenstrom (mAs) von entscheidender Bedeutung. 120-kVp-Protokolle werden immer noch am häufigsten an Geräten eingesetzt, wo der Röhrenstrom gerade bei adipöseren oder muskulöseren Patienten nicht ausreichend hochreguliert werden kann, um mit $100 \mathrm{kVp}$ zu scannen. 80-kVp-Protokolle sind aufgrund des Bildrauschens bei älteren CT-Scannern dann häufig

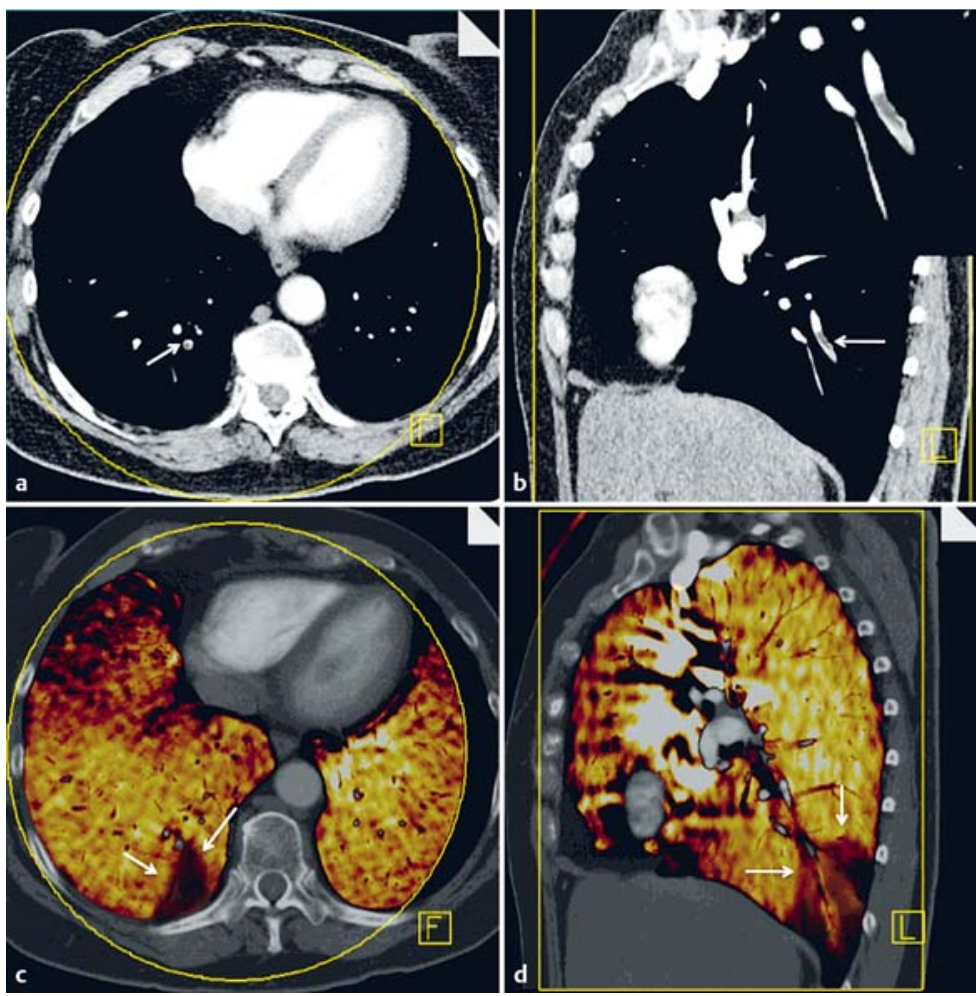

Abb. 4 Patient mit segmentaler Lungenembolie im Segment 10 rechts mittels DualEnergy-Technik untersucht. a, b Axiale (a) und sagittale (b) „mixed images“, die den Datensätzen der konventionellen Single-Energy-Technik vom Bildeindruck her entsprechen. c, d Die gleichen axialen (c) und sagittalen (d) Schichten zeigen in den jodselektiven Rekonstruktionen den Nachweis eines pleuraständigen keilförmigen Perfusionsdefektareals im Versorgungsgebiet der betroffenen Segmentarterie. gar nicht diagnostisch einsetzbar, wohingegen die Röhrenspannung bei neuen Scannergenerationen für ein breites Patientenspektrum bis auf $70 \mathrm{kVp}$ reduziert werden kann [18,19]. 100-kVp-Protokolle können bei Patienten bis $125 \mathrm{kgKG}$ ohne Einbußen bei der diagnostischen Sicherheit zum Einsatz kommen [20]. Der entscheidende Vorteil der Reduktion der Röhrenspannung liegt neben der niedrigeren Strahlendosis in einer signifikanten Steigerung des Jodkontrastes, da es durch eine Absenkung der Spannung zu einer Verschiebung des Photonenspektrums in die Nähe der K-Kante von Jod (33 keV) kommt. Hierdurch kann man bei gleichbleibender Kontrastmittelmenge ein relativ höheres Enhancement erzielen oder die Gesamtmenge an appliziertem Kontrastmittel verringern [18, 19,21,22].

\section{- Postprocessing}

Schichtdicke, Inkrement. Zur sicheren Beurteilung sind dünn rekonstruierte Schichten unabdingbar; 0,6-1,5 mm Schichtdicke mit einem Inkrement von 0,8 oder $20 \%$ Überlappung werden zur primären Befundung empfohlen. Dreidimensionale multiplanare Reformationen (MPRs) sollten wie bei der Beurteilung von Gefäßen generell zusätzlich zu den axialen Schichten in der Gefäßebene zurate gezogen werden.

Dual-Energy-Technik. Neuere Untersuchungstechniken wie die Dual-Energy-Technik und die damit verbundene Möglichkeit der Rekonstruktion von virtuell monoenergetischen Datensätzen mit Spannungen bis $40 \mathrm{keV}$ versprechen eine Option, a posteriori die Kontrastierung zu verbessern oder Kontrastmittel beim Scan einzusparen [23 - 25]. Hauptsächlich aber bietet die Dual-Energy-Technik die Möglichkeit, neben der Erzeugung der gewohnten morphologischen Datensätze jodselektive Datensätze zu erstellen, die im Parenchym die Lungenperfusion zum Zeitpunkt der Bildakquisition abbilden (sog. Snap-Shot-PerfusionsMaps, Abb. 4c, d).

Jodverteilungskarten für die Gefäße können ebenfalls erzeugt werden und stellen dann Jod selektiv und farblich codiert bis in die subsegmentalen kleinen Gefäßen dar, was zum einen die Diagnostik isolierter subsegmentaler Embolien erleichtern kann, aber auch bei Obstruktion-Score-Berechnungen hilfreich ist.

Mit der Dual-Energy-CT ist es auch möglich, Xenon selektiv darzustellen. Erste Arbeiten zur Perfusionsund Ventilationsbildgebung mit Dual-Energy-CT konnten bereits aufzeigen, dass hierdurch Diskrepanzen zwischen Lungenventilation und Perfusion vereinfacht visualisiert werden können. 


\section{Kontrastmittelgabe}

Jodflussrate. Ein suffizientes vaskuläres Enhancement wird durch eine ausreichende Kontrastmittelgabe und eine ausreichende, an die Scandauer angepasste Injektionsgeschwindigkeit erreicht. Der entscheidende Parameter für eine erfolgreiche CT-Angiografie ist die Jodflussrate, definiert durch die Jodmenge, die pro Sekunde injiziert wird (,iodine delivery rate“, IDR) [26]. Dieser Parameter wird wie folgt berechnet:

- Jodkonzentration des Kontrastmittels multipliziert mit der Flussgeschwindigkeit, mit der das Kontrastmittel appliziert wird

- Beispiel: $400 \mathrm{mg} \mathrm{Jod} / \mathrm{ml}$ (=0,4g Jod $/ \mathrm{ml})$ und $4,0 \mathrm{ml} / \mathrm{s}$ IDR $=0,4 \mathrm{~g} \mathrm{Jod} / \mathrm{ml} \times 4,0 \mathrm{ml} / \mathrm{s}=1,6 \mathrm{~g} \mathrm{Jod} / \mathrm{s}$

In der Regel sind Jodflussraten zwischen $1,5 \mathrm{~g} \mathrm{Jod} / \mathrm{s}$ und $1,8 \mathrm{~g} \mathrm{Jod} / \mathrm{s}$ ausreichend.

Artefaktreduktion. Wichtig ist neben dem Anwärmen des Kontrastmittels auf Körpertemperatur (zur Verminderung der Viskosität) auch die dem Kontrastmittelbolus folgende Injektion eines Kochsalz-Chasers, um den „Totraum“ von ca. $30 \mathrm{ml}$ zwischen Injektionsstelle und rechtem Herzen zu spülen. Hierdurch werden Artefakte in der V. cava superior minimiert, die besonders die Beurteilung der rechten Pulmonalarterie einschränken können. Besonders für Dual-Energy-CTProtokolle wird zusätzlich eine kaudokraniale Scanrichtung empfohlen, um in den Lungenoberfeldern hochkontrastartefaktbedingte artifizielle „Perfusionsdefekte“ durch die V. cava superior zu reduzieren.

Kontrastmittelmenge. Die Menge an für CTPA-Untersuchungen appliziertem Kontrastmittel hängt neben der angestrebten IDR gerade bei älteren Scannern auch entscheidend von der Scandauer ab. Da moderne Scanner nur noch wenige Sekunden für den Thoraxscan auch bei dünner Kollimationswahl benötigen, konnte die Menge in den letzten Jahren stetig reduziert werden: Bei einer Scandauer von 20 Sekunden werden $100 \mathrm{ml}$ Kontrastmittel empfohlen, bei Scanzeiten unter 5 Sekunden sind lediglich noch ca. 50-70 ml notwendig [27]. Wie bereits eingangs erwähnt, kann die Kontrastmittelmenge mit modernen Scannern, niedrigerer Röhrenspannung und kV-Einstellungen und neuen Techniken wie virtuell niedrigmonoenergetischen Rekonstruktionen weiter reduziert werden [23], wobei diese Verfahren nicht an allen CT-Systemen einsetzbar sind (Tab.2). Ein Nachteil von High-Pitch-CTPA-Untersuchungen mit sehr wenig Kontrastmittel liegt auch darin, dass häufig lediglich sog. Single-Rule-OutUntersuchungen entstehen, die keine suffiziente Beurteilung der Aorta mehr erlauben (Abb.5).

\section{Checkliste}

Rekonstruktionen für die Befundung

- 0,6-1,5 mm Schichtdicke

- Inkrement 0,8/20\% Overlap

- verdächtige Kontrastmittelaussparungen intravasal in 3D-MPR anschauen $\rightarrow$ Reduktion falsch positiver Ergebnisse

- falls Scan in Dual-Energy-Technik erfolgt, Rekonstruktion von jodselektiven Perfusionskarten sowie Rekonstruktion von virtuellen niedermonoenergetischen Datensätzen

Anpassung an das Gewicht. Idealerweise wird die Menge des Kontrastmittels genauso wie die Wahl von Röhrenspannung und Röhrenstrom an das Gewicht des Patienten angepasst [28].

Kontrastmittel-Timing. Um auf die patientenspezifische Zirkulationszeit, die maßgeblich mit der kardialen Auswurfleistung zusammenhängt, adäquat reagieren zu können, werden 2 verschiedene Verfahren des Kontrastmittel-Timings verwendet: das Testbolusverfahren [29] oder das Bolus-Tracking. Um bei neueren CT-Systemen zusätzlich die arteriellen und linksventrikulären Strukturen beurteilen zu können, kann das Split-Bolus-Verfahren eingesetzt werden [30].

EKG-Triggering. Studien haben gezeigt, dass die Ausrichtung der CTPA am EKG bei ähnlicher Kontrastmittelmenge das pulmonale Enhancement verbessert und gleichzeitig Bewegungsartefakte verringert [31]. Neben der Anwendung in Studien wird das EKG-Triggering bei der CTPA jedoch aktuell nicht routinemäßig eingesetzt.

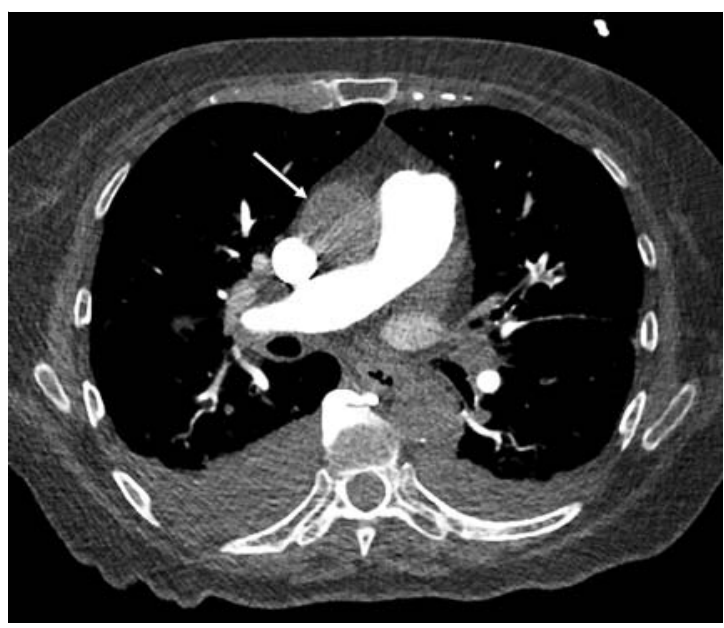

Abb. 5 Das Problem des „SingleRule-Out-Protokolls" bei HighPitch-Untersuchungen mit Low-DoseKontrastmittelansätzen ist die insuffiziente Kontrastierung der aortalen Gefäßabschnitte. 
Tabelle 2

Beispielprotokolle für CTPA-Untersuchungen mit verschiedenen CT-Systemen.

\begin{tabular}{|c|c|c|c|}
\hline Parameter & $\begin{array}{l}\text { Triple-Rule-Out } \\
\text { CT-Angiografie des Thorax }\end{array}$ & Dual-Energy-СTPA & Single-Energy-СТPA \\
\hline Gerätehersteller & $\begin{array}{l}\text { Siemens Healthineers, } \\
\text { Somatom Flash }\end{array}$ & $\begin{array}{l}\text { Siemens Healthineers, } \\
\text { Somatom Definition }\end{array}$ & $\begin{array}{l}\text { Siemens Healthineers, } \\
\text { Somatom Emotion }\end{array}$ \\
\hline MDCT & $2 \times 128$ & $2 \times 64$ & $1 \times 16$ \\
\hline Kollimation (mm) & 0,6 & 1,2 & 0,6 \\
\hline EKG-Triggering & ja & nein & nein \\
\hline Literatur & [32] & [24] & [33] \\
\hline benötigter venöser Zugang & $18 \mathrm{G}$ & $18 \mathrm{G}$ & $20 \mathrm{G}$ \\
\hline Lage „region of interest“ & Aorta ascendens & A. pulmonalis & A. pulmonalis \\
\hline Scan-Richtung & kraniokaudal & kaudokranial & kraniokaudal \\
\hline Röhrenspannung (kV) & 100 & $140 / 80$ & $100-130$ \\
\hline HU-Schwellenwert & $100 \mathrm{HU}$ & $100 \mathrm{HU}$ & $80 \mathrm{HU}$ \\
\hline Kontrastmittelkonzentration $(\mathrm{mg} / \mathrm{ml})$ & 370 & 400 & 400 \\
\hline Kontrastmittelfluss (ml/s) & 6 & 4 & 4 \\
\hline Kontrastmittelmenge (ml) & 90 & 100 & 100 \\
\hline $\mathrm{NaCl}$-Fluss ( $\mathrm{ml} / \mathrm{s})$ & 6 & 4 & 4,0 \\
\hline $\mathrm{NaCl}$-Menge (ml) & 30 & 50 & 30 \\
\hline IDR (g Jod/s) & 2,22 & 1,6 & 1,6 \\
\hline
\end{tabular}

\section{Atemkommandos}

Problem der zu tiefen Inspiration. Durch eine zu tiefe Inspiration besteht bei CTPA-Untersuchungen trotz suffizientem venösem Zugang und suffizienter Flussgeschwindigkeit des applizierten Kontrastmittels die Gefahr einer nicht diagnostischen Untersuchung. Die physiologische Erklärung dafür ist, dass bei einer tiefen Inspiration Blut aus der V. cava inferior angesaugt wird (sog. „abdomino-thoracic pump“). Dieses Blut ist nicht mit Kontrastmittel gesättigt und führt so zu einer Unterbrechung der Kontrastmittelsäule. Darüber hinaus kann ein Valsalva-Manöver zu einer insuffizienten Kontrastierung führen. Dies lässt sich durch den Anstieg des intrathorakalen Drucks erklären, der den Fluss in der V. cava superior und damit auch den Kontrastmittelstrom zum Herz vermindert.
Geschwindigkeit des Systems. Bei sehr schnellen Untersuchungstechniken („ultra-high pitch“ von 2,83,2) sind keine Atemkommandos notwendig, da Bewegungsartefakte durch die schnelle Bildakquisition keine Rolle mehr spielen. Bei langsameren CT-Systemen wird von manchen Studien eine Aufnahme in Exspiration vorgeschlagen. Die Diagnostik der basalen Lunge ist dann jedoch durch Dystelektasen verändert. Außerdem ist der Atemanhalt in Exspiration anstrengender und wird besonders von Patienten mit Dyspnoe nur kurz bis gar nicht toleriert und meist insuffizient ausgeführt. Unserer Erfahrung nach hat sich für die CTPA das Atemkommando „Sanft einatmen - Luft anhalten“ als ideal herausgestellt. 


\section{Rechtsherzbelastung}

Die Zusatzdiagnose einer Rechtsherzbelastung (,right ventricular dysfunction“, RVD) durch die morphologische Beurteilung des rechten Ventrikels im Rahmen von CTPA-Untersuchungen spielt eine wichtige Rolle besonders für die Prognose des Patienten.

RV/LV-Ratio. Das Verhältnis von maximaler Weite der rechten zur linken Kammer, gemessen auf der axialen Schicht mit dem maximalen Durchmesser, ist ein einfach zu ermittelnder Parameter. Hierbei ist wichtig zu erwähnen, das die maximale Weite der Ventrikel auf den axialen Schichten nicht zwangsläufig für den linken und rechten Ventrikel auf einer Schicht liegen müssen. Der Schwellenwert liegt bei der RV/LV-Ratio je nach Studie zwischen 0,9 und 1,2. Bei einem Wert $>1,2$ besteht der hochgradige Verdacht einer Rechtsherzbelastung (Abb.6). Zudem geht eine Ratio > 1,2 nachgewiesenermaßen mit einer erhöhten Mortalität einher [34].

Weitere Zeichen. Abweichend von der Methode der RV/LV-Ratio empfehlen andere Studien die Durchführung einer 3D-Volumetrie [35]. Weitere Zeichen einer Rechtsherzbelastung in der CTPA sind das „septal bowing“, eine paradoxe Vorwölbung der eigentlichen Niederdruckkammer des rechten Herzens in das linksventrikuläre Hochdrucksystem, sowie ein Nachweis von Kontrastmittelrückstau in die V. cava inferior und die Lebervenen.

\section{Tipps und Tricks}

Atemkommando

- wenn verfügbar, Ultra-HighPitch-Protokolle verwenden, um Bewegungsartefakte durch Atmung „auszuschalten“

- Daten nur in Exspiration akquirieren, wenn patientenseitig wahrscheinlich durchführbar,

- bei einer Datenakquisition in Inspiration sanfte Inspiration anstreben

- um ein Valsavamanöver und die „abdomino-thoracic pump“ zu vermeiden, Atemkommandos vorher mit dem Patienten üben! sonst gilt: besser eine gehaltene Inspirationsaufnahme als eine veratmete Exspirationsaufnahme

\section{Atypische Lungenembolien}

Fettembolien, Luftembolien, Fruchtwasserembolien und Fremdkörperembolien (Abb.7) sind weitere seltene emboligene Ursachen einer LE, und ebenfalls mit der CTPA diagnostizierbar, sind jedoch außerhalb des Fokus dieser Übersichtsarbeit [36].
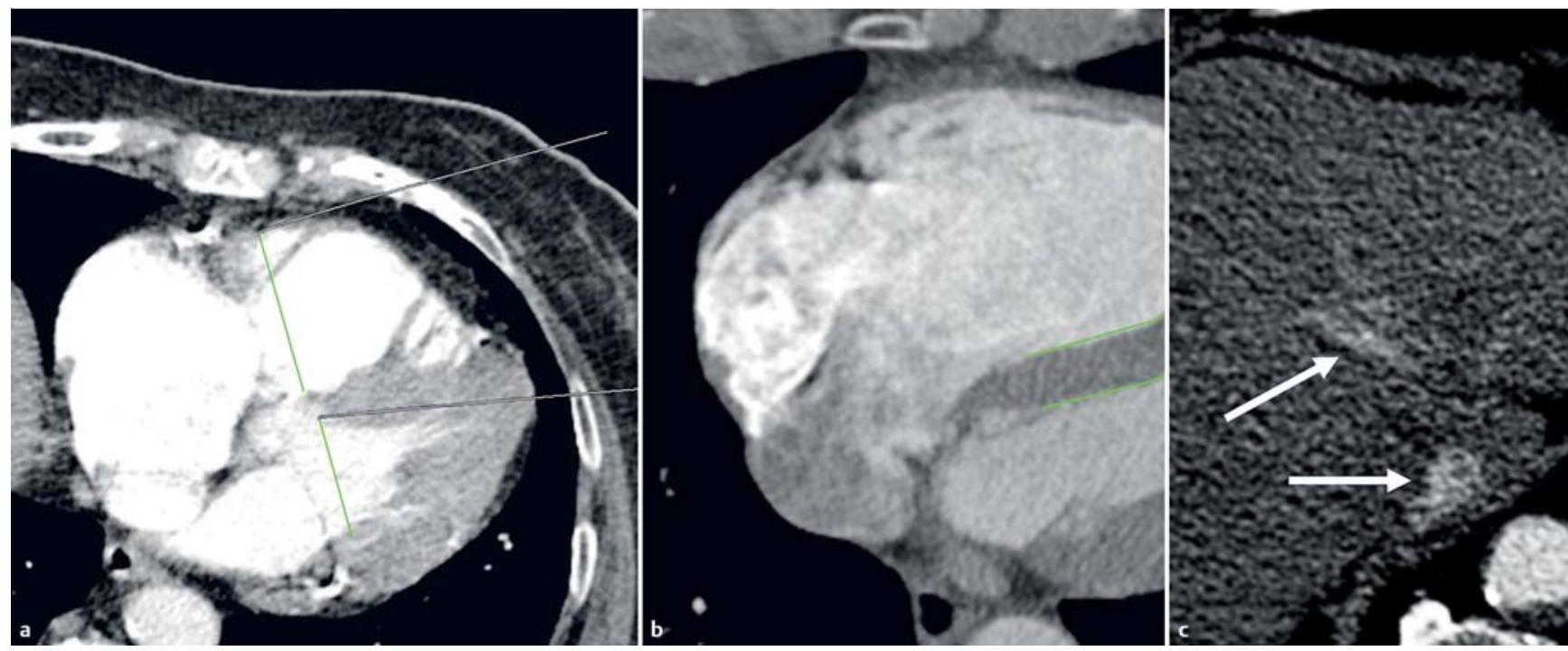

Abb. 6 Zeichen einer Rechtsherzbelastung. a Pathologisch erhöhte RV/LV-Ratio > 1,2. b Paradoxe Septumbewegung in Richtung des linken Ventrikels. c Reflux von Kontrastmittel in die V. cava inferior und die Lebervenen. 
Abb. 7 Katheterfragmentembolie bei einem Kleinkind in eine Segmentarterie des rechten Mittellappens.

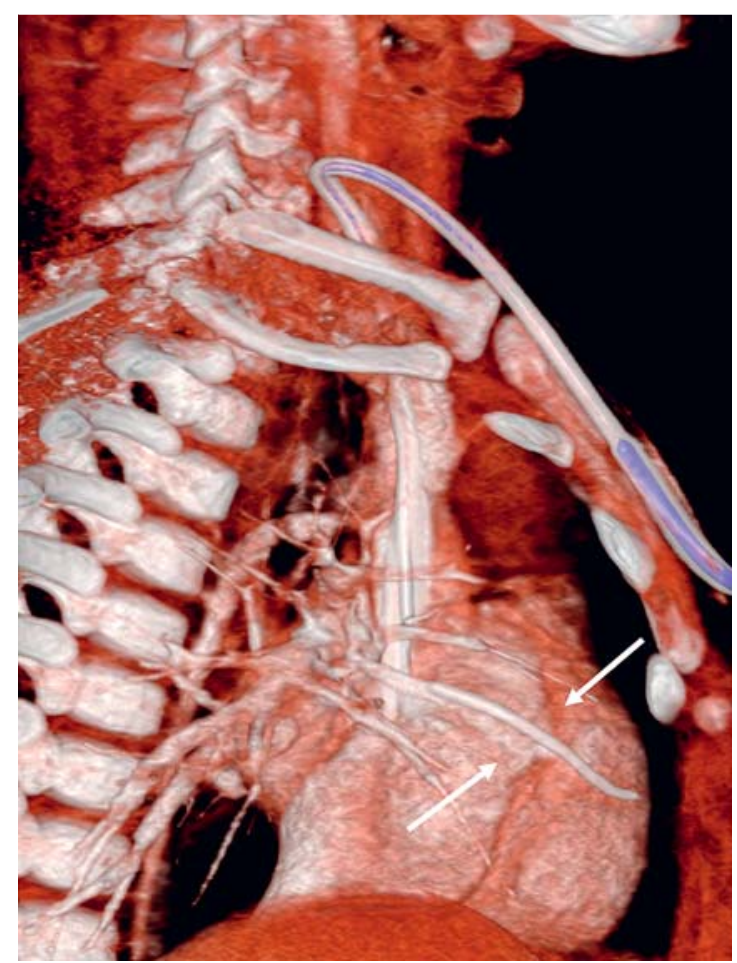

\section{Tipps und Tricks}

Welche Informationen interessieren den Kliniker im Befund einer CTPA zum Ausschluss einer Lungenembolie?

Bildqualität

- allgemeine Aussage zur Bildqualität zur Einschätzung der diagnostischen Sicherheit (z. B. Atemartefakte, insuffiziente Kontrastierung, auch evtl. fehlgesetzte „region of interest“ für Bolus-Triggering in der Aorta oder Ähnliches beschreiben)

- Beschreibung von Artefakten (z.B. Hochkontrastartefakte durch ICD oder Schrittmacheraggregate, Klappenersatz, Kontrastmitteleinstrom in der V. cava superior)

Embolie

- Handelt es sich um eine zentrale, segmentale oder isoliert subsegmentale LE?

- CT-morphologische Beschreibung der Emboli (z. B. okklu- dierend, partiell okkludierend, randständig umspült, „webs and bands" als Zeichen der Reorganisation)

Rechtsherzbelastungszeichen

- pathologisch erhöhte RV/LV-Verhältnisse

- Rückstau von Kontrastmittel in Lebervenen und V. cava inferior, „septal bowing“

Weitere Merkmale

- Lungeninfarkte (falls ja, Anhalt für eine Superinfektion im Sinne von Postinfarktpneumonien)?

- Zeichen einer CTEPH (außerhalb des Fokus dieses Übersichtsartikels)?

Differenzialdiagnosen bei Ausschluss einer LE

\section{LE-Diagnostik bei Schwangeren}

Die LE ist die häufigste Todesursache bei Schwangeren. Schwierigkeiten bereiten - noch mehr als beim normalen Patientenkollektiv - häufig die unspezifischen Symptome, die sich bei Schwangeren oft einreihen in eine ganze Anzahl neu auftretender körperlicher Veränderungen und Beschwerden.

D-Dimer-Test. Der D-Dimer-Test ist bei Schwangeren i.d.R. positiv. Ansätze, neue Grenzwerte für die Schwangerschaft zu etablieren [37], haben sich bisher nicht flächendeckend durchgesetzt. Ein negativer D-Dimer-Test schließt jedoch bei Schwangeren eine LE ebenso zuverlässig aus wie bei anderen Patientengruppen [1].

Ein negativer D-Dimer-Test schließt auch bei Schwangeren die LE aus und macht eine weitere Diagnostik überflüssig. Ein positiver D-Dimer-Test kann jedoch in der Schwangerschaft regelhaft physiologischerweise vorkommen.

Sonografie. Als erste diagnostische Maßnahme wird eine Ultraschalluntersuchung der Becken- und Beinvenen durchgeführt, da bei einer TVT eine Behandlung sowieso indiziert ist und man somit keine weitere Bildgebung zur Therapieentscheidung durchführen muss [1].

Szintigrafie oder CTPA. Ist die Ultraschalluntersuchung unauffällig oder nicht eindeutig und besteht weiterhin der Verdacht auf eine LE, kommen für die weitere Diagnostik - wenn beide Modalitäten verfügbar sind eine Szintigrafie oder eine CTPA infrage. Bei beiden Verfahren ist die fetale Exposition und die Strahlenbelastung der Mutter - bei der Lungenemboliediagnostik besonders die der Brustdrüsen - zu beachten. Die Strahlenbelastung des Feten ist bei beiden Verfahren sehr niedrig (CTPA 0,003-0,13 mSv, Szintigrafie 0,060,12 mSv). Zur Reduktion der Strahlenbelastung der Mutter wurde früher häufig zunächst die Szintigrafie bevorzugt, mit den neuen CT-Scannern und den technischen Entwicklungen zur Dosisreduktion fällt dieses Argument weg [38].

Szintigrafie. Kommt die Szintigrafie zum Einsatz, wird meist zunächst eine Perfusionsszintigrafie durchgeführt, an die sich ggf. eine Ventilationsszintigrafie anschließt. Eine nicht diagnostische Szintigrafie sollte um eine CTPA ergänzt werden. Das American College of Radiology (ACR) empfiehlt in den Leitlinien von 2012 eine Perfusionsszintigrafie mit Reduktion des Radio- 
pharmazeutikums um den Faktor 2 oder mehr mit höheren Akquisitionszeiten, aber de-facto reduzierter Strahlendosis [39]. Bei einem normalen Perfusionsscan kann die Szintigrafie laut ACR abgebrochen werden, wenn eine konventionelle Röntgenthorax-Aufnahme zuvor ebenfalls unauffällig war.

Jodhaltiges Kontrastmittel. Ist eine kontrastverstärkte Untersuchung indiziert, kann der Einsatz jodhaltigen Kontrastmittels in der Schwangerschaft als sicher angesehen werden [40]. Die Gabe eines jodhaltigen Kontrastmittels während der Schwangerschaft kann aber zu Schilddrüsenfunktionsstörungen beim Kind führen, weshalb die Schilddrüsenfunktion in diesem Fall in der 1.Woche nach der Geburt laborchemisch geprüft werden sollte. Mütter können nach der Applikation von jodhaltigem Kontrastmittel unverändert weiter stillen [41].

\section{Über die Autoren}

\section{Sonja Sudarski}

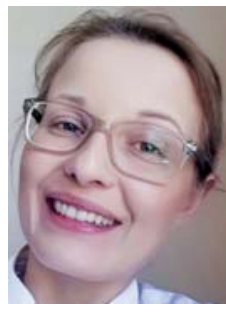

Dr. med. 2007 - 2013 Studium der Humanmedizin an der Medizinischen Fakultät Mannheim der Universität Heidelberg. 2014 Promotion. Seit 2013 Assistenzärztin am Institut für Klinische Radiologie und Nuklearmedizin. Forschungsschwerpunkt: quantitative Radiologie in der kardiothorakalen und onkologischen Bildgebung.

\section{Thomas Henzler}

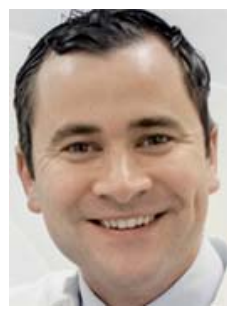

Prof. Dr. med. 2001 - 2007 Studium der Humanmedizin an den Universitäten Ulm und Bern. 2007 Promotion. 2008 - 2010 Assistenzarzt am Institut für Klinische Radiologie und Nuklearmedizin der Universitätsmedizin Mannheim. 2012 Habilitation. 2013 Facharztanerkennung Radiologie. Seit 2012 Oberarzt am Institut für Klinische Radiologie und Nuklearmedizin der Universitätsmedizin Mannheim. Leiter des Geschäftsfelds Kardiothorakale Bildgebung und Computertomografie. Forschungsschwerpunkt: Weiterentwicklung neuartiger CT-Techniken im Bereich der funktionellen kardiothorakalen und onkologischen Bildgebung.

\section{Kernaussagen}

- Die CTPA ist der Goldstandard der nicht invasiven Diagnostik der LE und liefert wichtige Zusatzinformationen wie das Vorliegen einer Rechtsherzbelastung.

- Die Revisionen der ESC-Leitlinien stärken im Vergleich von 2008 zu 2014 die Position der CTPA im Diagnosealgorithmus weiter.

- Niedrigere kV-Werte sind bei der CTPA im Hinblick auf eine geringere Strahlenbelastung und ein stärkeres Kontrastmittelenhancement zu bevorzugen, falls dies scannertechnisch umsetzbar ist.

- Neue technische Möglichkeiten wie z. B. die Dual-Energy-Technik oder das Ultra-High-Pitch-Verfahren können die Bildqualität und somit die Diagnostik weiter verbessern - sind jedoch noch nicht weit verbreitet.

- Kontrastmittelprotokolle und Atemkommandos sind die 2 Hauptfehlerquellen bei nicht diagnostischen CTPAs.

- Bei Schwangeren mit Verdacht auf eine LE sollte bei initial erfolglosem Ausschluss einer TVT mittels Ultraschall frühzeitig die weitere Bildgebung mittels CTPA (oder zentrenabhängig auch Szintigrafie) zum Einsatz kommen.

\section{Abstract}

Pulmonary embolism (PE) requires a quick diagnostic algorithm, as the untreated disease has a high mortality and morbidity. Crucial for the diagnostic assessment chosen is the initial clinical likelihood of PE and the individual risk profile of the patient. The overall goal is to diagnose or rule out PE as quickly and safely as possible or to initiate timely treatment if necessary. CT angiography of the pulmonary arteries (CTPA) with multislice CT scanner systems presents the actual diagnostic reference standard. With CTPA further important diagnoses can be made, like presence of right ventricular dysfunction. There are different scan and contrast application protocols that can be applied in order to gain diagnostic examinations with sufficient contrast material enhancement in the pulmonary arteries while avoiding all kinds of artifacts. This review article is meant to be a practical guide to examine patients with suspected PE according to the actual guidelines.

\section{Keywords}

Pulmonary embolism - CT pulmonary angiography . right ventricular dysfunction - diagnostic algorithms . protocols 


\section{Korrespondenzadresse}

Dr. med. Sonja Sudarski

Institut für Klinische Radiologie und Nuklearmedizin

Universitätsmedizin Mannheim

Medizinische Fakultät Mannheim

Universität Heidelberg

Theodor-Kutzer-Ufer 1-3

68167 Mannheim

Tel. +49 $621383-2067$

Fax: +49 621 383-1910

E-Mail: sonja.sudarski@medma.uni-heidelberg.de

Interessenkonflikt: kein Interessenkonflikt angegeben

\section{Literatur}

1 Konstantinides SV, Torbicki A, Agnelli G et al. 2014 esc guidelines on the diagnosis and management of acute pulmonary embolism. Eur Heart J 2014; 35: 3033-3069, 69a-69k

2 Pengo V, Lensing AW, Prins $\mathrm{MH}$ et al. Incidence of chronic thromboembolic pulmonary hypertension after pulmonary embolism. N Engl ] Med 2004; 350: 2257 - 2264

3 Righini M, Van Es J, Den Exter PL et al. Age-adjusted d-dimer cutoff levels to rule out pulmonary embolism: The adjust-pe study. JAMA 2014; 311: 1117-1124

4 Engelberger RP, Kucher N. Ultrasound-assisted thrombolysis for acute pulmonary embolism: A systematic review. Eur Heart J 2014; 35: 758-764

5 van Belle A, Buller HR, Huisman MV et al. Effectiveness of managing suspected pulmonary embolism using an algorithm combining clinical probability, d-dimer testing, and computed tomography. JAMA 2006; 295: $172-179$

6 Anderson DR, Kahn SR, Rodger MA et al. Computed tomographic pulmonary angiography vs ventilation-perfusion lung scanning in patients with suspected pulmonary embolism: A randomized controlled trial. JAMA 2007; 298: 2743-2753

7 Righini M, Le Gal G, Aujesky D et al. Diagnosis of pulmonary embolism by multidetector ct alone or combined with venous ultrasonography of the leg: A randomised non-inferiority trial. Lancet 2008; 371: $1343-1352$

8 Stein PD, Fowler SE, Goodman LR et al. Multidetector computed tomography for acute pulmonary embolism. N Engl J Med 2006; 354: 2317-2327

9 Sostman HD, Stein PD, Gottschalk A et al. Acute pulmonary embolism: Sensitivity and specificity of ventilation-perfusion scintigraphy in pioped ii study. Radiology 2008; 246: 941 946

10 Schembri GP, Miller AE, Smart R. Radiation dosimetry and safety issues in the investigation of pulmonary embolism. Semin Nucl Med 2010; 40: 442-454

11 Schiebler ML, Nagle SK, Francois C] et al. Effectiveness of mr angiography for the primary diagnosis of acute pulmonary embolism: Clinical outcomes at 3 months and 1 year. J Magn Reson Imaging 2013; 38: 914-925
12 Adhikari NK, Fowler RA, Bhagwanjee S et al. Critical care and the global burden of critical illness in adults. Lancet 2010; 376: $1339-1346$

13 Araoz PA, Gotway MB, Harrington JR et al. Pulmonary embolism: Prognostic ct findings. Radiology 2007; 242: 889-897

14 Henzler T, Roeger S, Meyer M et al. Pulmonary embolism: CT signs and cardiac biomarkers for predicting right ventricular dysfunction. Eur Respir J 2012; 39: 919-926

15 Meinel FG, Nance JWJr, Schoepf UJ et al. Predictive value of computed tomography in acute pulmonary embolism: Systematic review and meta-analysis. Am J Med 2015; 128: 747 59 e2

16 Carrier M, Righini M, Wells PS et al. Subsegmental pulmonary embolism diagnosed by computed tomography: Incidence and clinical implications. A systematic review and meta-analysis of the management outcome studies. J Thromb Haemost 2010; 8: $1716-1722$

17 Stein PD, Goodman LR, Hull RD et al. Diagnosis and management of isolated subsegmental pulmonary embolism: Review and assessment of the options. Clin Appl Thromb Hemost 2012; $18: 20-26$

18 Szucs-Farkas Z, Christe A, Megyeri B et al. Diagnostic accuracy of computed tomography pulmonary angiography with reduced radiation and contrast material dose: A prospective randomized clinical trial. Invest Radiol 2014; 49: 201 - 208

19 Szucs-Farkas Z, Schaller C, Bensler S et al. Detection of pulmonary emboli with ct angiography at reduced radiation exposure and contrast material volume: Comparison of $80 \mathrm{kvp}$ and 120 kvp protocols in a matched cohort. Invest Radiol 2009; 44: 793 - 799

20 Megyeri B, Christe A, Schindera ST et al. Diagnostic confidence and image quality of ct pulmonary angiography at 100 kvp in overweight and obese patients. Clin Radiol 2015; 70: $54-61$

21 Hansmann J, Fink C, Jost $G$ et al. Impact of iodine delivery rate with varying flow rates on image quality in dual-energy ct of patients with suspected pulmonary embolism. Acad Radiol 2013; 20: 962 - 971

22 Szucs-Farkas Z, Schibler F, Cullmann J et al. Diagnostic accuracy of pulmonary ct angiography at low tube voltage: Intraindividual comparison of a normal-dose protocol at $120 \mathrm{kvp}$ and a low-dose protocol at $80 \mathrm{kvp}$ using reduced amount of contrast medium in a simulation study. AJR Am J Roentgenol 2011; 197: W852-W859

23 Delesalle MA, Pontana F, Duhamel A et al. Spectral optimization of chest ct angiography with reduced iodine load: Experience in 80 patients evaluated with dual-source, dual-energy ct. Radiology 2013; 267: 256-266

24 Apfaltrer P, Bachmann V, Meyer M et al. Prognostic value of perfusion defect volume at dual energy cta in patients with pulmonary embolism: Correlation with cta obstruction scores, ct parameters of right ventricular dysfunction and adverse clinical outcome. Eur J Radiol 2012; 81: 3592 - 3597

25 Meyer M, Haubenreisser $\mathrm{H}$, Sudarski S et al. Where do we stand?. Functional imaging in acute and chronic pulmonary embolism with state-of-the-art ct Eur J Radiol 2015; 84 : $2432-2437$

26 Heusch P, Lanzman RS, Aissa J et al. Evaluation of a high iodine delivery rate in combination with low tube current for dose 
reduction in pulmonary computed tomography angiography. J Thorac Imaging 2014; 29: 293-297

27 Hartmann IJ, Wittenberg R, Schaefer-Prokop C. Imaging of acute pulmonary embolism using multi-detector ct angiography: An update on imaging technique and interpretation. Eur J Radiol 2010; 74: 40 - 49

28 Bae KT. Intravenous contrast medium administration and scan timing at ct: Considerations and approaches. Radiology 2010; 256: $32-61$

29 Henzler T, Meyer M, Reichert M et al. Dual-energy ct angiography of the lungs: Comparison of test bolus and bolus tracking techniques for the determination of scan delay. Eur J Radiol 2012; 81: 132 - 138

30 Cornea AM, McCullough BJ, Mitsumori LM et al. Enhancement of the pulmonary arteries and thoracic aorta: Comparison of a biphasic contrast injection and fixed delay protocol with a monophasic injection and a timing bolus protocol. Emerg Radiol 2015; 22: $231-237$

31 Bolen MA, Renapurkar RD, Popovic ZB et al. High-pitch ecgsynchronized pulmonary ct angiography versus standard ct pulmonary angiography: A prospective randomized study. AJR Am J Roentgenol 2013; 201: 971 - 976

32 Bamberg F, Marcus R, Sommer W et al. Diagnostic image quality of a comprehensive high-pitch dual-spiral cardiothoracic ct protocol in patients with undifferentiated acute chest pain. Eur J Radiol 2012; 81: 3697 - 3702

33 Apfaltrer P, Henzler T, Meyer M et al. Correlation of ct angiographic pulmonary artery obstruction scores with right ventricular dysfunction and clinical outcome in patients with acute pulmonary embolism. Eur J Radiol 2012; 81: 2867 2871
34 Furlan A, Aghayev A, Chang CC et al. Short-term mortality in acute pulmonary embolism: Clot burden and signs of right heart dysfunction at ct pulmonary angiography. Radiology 2012; 265: 283-293

35 Kang DK, Thilo C, Schoepf UJ et al. Ct signs of right ventricular dysfunction: Prognostic role in acute pulmonary embolism. JACC Cardiovasc Imaging 2011; 4: 841 - 849

36 Bach AG, Restrepo CS, Abbas J et al. Imaging of nonthrombotic pulmonary embolism: Biological materials, nonbiological materials, and foreign bodies. Eur J Radiol 2013; 82: e120e141

37 Morse M. Establishing a normal range for $\mathrm{d}$-dimer levels through pregnancy to aid in the diagnosis of pulmonary embolism and deep vein thrombosis. J Thromb Haemost 2004; 2: $1202-1204$

38 Weisser G, Steil V, Neff KW et al. radiology and pregnancy: Part 2: Clinical recommendations. Radiologe 2013; 53: 75-82; quiz 3-4

39 Bettmann MA, Baginski SG, White RD et al. Acr appropriateness criteria(r) acute chest pain - suspected pulmonary embolism. J Thorac Imaging 2012; 27: W28-W31

40 Webb JA, Thomsen HS, Morcos SK. Members of Contrast Media Safety Committee of European Society of Urogenital R. The use of iodinated and gadolinium contrast media during pregnancy and lactation. Eur Radiol 2005; 15: $1234-1240$

41 Baert A, Knauth M, Thomsen HS et al. Contrast media: Safety issues and esur guidelines. Berlin, Heidelberg: Springer; 2009 


\section{CME-Fragen}

Die folgenden Fragen beziehen sich auf den vorangehenden Beitrag. Bitte schicken Sie uns die entsprechenden Lösungsbuchstaben. Jeweils eine Antwort ist richtig. Die Vergabe von CME-Punkten ist an die korrekte Beantwortung der Multiple-Choice-Fragen gebunden.

\section{1}

Welche Einteilung wird nach den aktuellen ESC-Leitlinien angewendet, um bei Patienten mit Verdacht auf eine Lungenembolie eine erste klinische Risikoeinschätzung vorzunehmen?
A Unterscheidung in Verdacht auf zentrale, segmentale oder subsegmentale LE
B Unterscheidung in High-Risk-LE und Non-High-Risk-LE
C Unterscheidung in leichte, mittlere und schwere LE
D Unterscheidung in Patienten mit Medikamenten zur Antikoagulation in der laufenden Medikation und solchen ohne solche Medikamente
E Unterscheidung je nach Anzahl der betroffenen Lungensegmente in mono- oder multi- segmentale LE

\section{2}

Welche der folgenden Bildgebungsverfahren ist nach den aktuellen Leitlinien eine adäquate Alternative zur CTPA bei Schwangeren mit Verdacht auf Lungenembolie?
A Pulmonalisangiografie zum indirekten Embolusnachweis als Kontrastmittelaussparung
B MR-Angiografie zur Darstellung des tiefen Beinvenensystems
C Perfusionsszintigrafie zur Darstellung von LE-bedingten Perfusionsausfällen
D Linksherzkatheter zur Beurteilung der Linksherzbelastung
E CT-Thorax nativ zum Ausschluss infarkttypischer Infiltrate

\section{3}

Bei welchen der folgenden CT-morphologischen Befunde im kontrastmittelgestützten CT-Thorax zum Ausschluss einer LE unterscheidet sich das weitere diagnostische bzw. therapeutische Vorgehen voneinander?
A segmentale LE ohne Rechtsherzbelastung vs. zentrale LE ohne Rechtsherzbelastung
B segmentale LE mit RV/LV-Ratio von 1,6 vs. segmentale LE mit RV/LV-Ratio von 2,6
C zentrale LE mit RV/LV-Ratio von 1,6 vs. zentrale LE mit RV/LV-Ratio von 2,6
D segmentale LE mit positivem D-Dimer-Test vs. zentrale LE mit positivem D-Dimer-Test
E segmentale vs. isolierte subsegmentale LE

Bei welcher Patientengruppe mit Verdacht auf eine LE hätte das Ergebnis eines D-Dimer-Tests eine Relevanz im Sinne eines „change of management" für das weitere diagnostische Vorgehen?
A negativer D-Dimer-Test bei Patienten mit hoher Prätestwahrscheinlichkeit für eine LE
B positiver D-Dimer-Test bei Schwangeren
C negativer D-Dimer-Test bei Schwangeren
D positiver D-Dimer-Test bei Tumorpatienten
E positiver D-Dimer-Test bei Patienten mit Verdacht auf High-Risk-LE

\section{5}

Welcher der folgenden Faktoren zählt nicht zu den typischen Ursachen für nicht diagnostische Bilddaten oder eingeschränkte Bildqualität bei der Akquisition von LE-Ausschluss-CT-Protokollen?
A Atmung des Patienten, dadurch Bewegungsartefakte
B zu niedriger Fluss bei der Kontrastmittelapplikation
C Hochkontrastartefakte durch Kontrastmittel in der V. cava superior
D Übergewicht des Patienten
E falsche Positionierung der „region of interest“ beim Bolus-Tracking

Welche rekonstruierte Schichtdicke ist für die sichere Beurteilung einer CTPA immer mindestens zu fordern?
A $0,2 \mathrm{~mm}$
B $1,5 \mathrm{~mm}$
C $3 \mathrm{~mm}$
D $5 \mathrm{~mm}$
E $0,5 \mathrm{~mm}$ 


\section{CME-Fragen ungenateresenenoble}

Welcher der folgenden Faktoren ist kein unabhängiger Risikofaktor für das Auftreten einer LE?
A Übergewicht
B Tumorerkrankung
C Kontrazeptiva-Einnahme
D Immobilisation durch OP
E Z.n. TVT/LE in der Anamnese

\section{8}

Welche der folgenden Aussagen zur nuklearmedizinischen Diagnostik bei Verdacht auf LE ist richtig?
A Eine negative Ventilationsszintigrafie allein reicht in vielen Fällen für die Diagnosestellung bei Verdacht auf eine LE aus.

B Bei einem positiven Befund in der Perfusionsszintigrafie braucht die ergänzende Ventilationsszintigrafie nicht mehr akquiriert zu werden.

C Die V/P-Szintigrafie ist eine sehr spezifische, aber wenig sensitive Methode.

D Die Szintigrafie ist aufgrund der niedrigen Strahlenbelastung die Methode der Wahl bei Verdacht auf eine LE.

E Die Szintigrafie ist aufgrund der erhöhten Strahlenbelastung für den Feten absolut kontraindiziert in der Schwangerschaft.

\section{9}

Was ist ein Zeichen der akuten Rechtsherzbelastung in der CTPA?
A „atrial bowing“
B Rückstau von Kontrastmittel in die Lebervenen
C eine gemessene Ratio von 0,7 der maximalen Durchmesser des RV zum LV in axialen Schichten
D der Nachweis eines oder mehrerer "hampton humps“
E eine miterfasste Aorta descendens mit einem Durchmesser von $>4 \mathrm{~cm}$

\section{0}

Welche der folgenden Aussagen zur Kontrastmittelgabe ist richtig?
A Eine „iodine delivery rate“ von 1,2 gilt als ausreichend für die suffiziente Kontrastierung einer CTPA-Untersuchung.

B Ein Fluss von $4 \mathrm{ml} / \mathrm{s}$ ist mindestens notwendig, um bei einer Osmolarität des Kontrastmittels von $400 \mathrm{mg} / \mathrm{ml}$ eine „iodine delivery rate“ von mindestens 1,6 zu erreichen.

C Eine Kochsalzbolusgabe unmittelbar nach der Kontrastmittelapplikation dient ausschließlich dem Spülen des venösen Zugangs, um ein Verstopfen durch das visköse Kontrastmittel zu verhindern.

D Die Vermeidung von Hochkontrastartefakten in der V. cava superior wird durch die CTPAUntersuchung mittels Bolus-Tracking effektiv vermieden.

E Zur Vermeidung von Unterbrechungen in der Kontrastmittelsäule intravasal sollte die Untersuchung in tiefer Inspiration erfolgen.

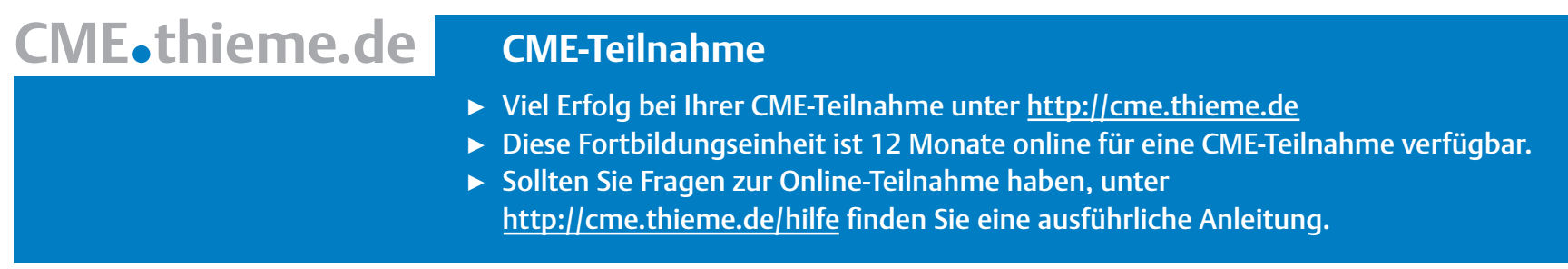

